\title{
El Malestar en el Empleo Temporal Involuntario
}

\section{Malaise in Involuntary Temporary Employment}

\author{
Josep M. Blanch y Leonor M. Cantera \\ Universidad Autónoma de Barcelona
}

\begin{abstract}
Resumen. El objetivo de este estudio fue analizar el papel de la voluntariedad de la situación ocupacional en el malestar psicológico en el empleo temporal. Se aplicó un cuestionario a una muestra de 463 personas incluidas en alguno de los siguientes cuatro tipos ideales de situación ocupacional: empleo estable, empleo temporal voluntario, empleo temporal involuntario y desempleo. Los resultados muestran diferencias significativas entre todos los tipos laborales en la escala de bienestar laboral. De mayor a menor puntuación media en esta escala, el orden de los tipos es: empleo estable, empleo temporal voluntario, desempleo y empleo temporal involuntario. Las respuestas textuales a las preguntas abiertas ponen de manifiesto un alto malestar en el estatus de empleo temporal involuntario, atribuido a la situación ocupacional presente y a la incertidumbre e inseguridad que genera el propio futuro laboral percibido.
\end{abstract}

Palabras clave: inseguridad laboral, bienestar, empleo temporal involuntario, malestar.

\begin{abstract}
The aim of this study was to analyze the occupational situation voluntary on the psychological malaise in temporary employment. A questionnaire was applied to a sample of 463 individuals included in one of the following four ideal types of occupational situation: stable employment, voluntary temporary employment, involuntary temporary employment, and unemployment. Results showed significant different among all occupational types in a scale of labor wellbeing. From higher to lower score in this scale, the ranking of the types is: stable employment, voluntary temporary employment, unemployment, and involuntary temporary employment. The responses to the open questions showed a high malaise in individuals in the condition of involuntary temporary employment, which is attributed to the current occupational situation and to the uncertainty and insecurity generated by the perceived labor future.

Key words: job insecurity, well-being, involuntary temporary employment, malaise.
\end{abstract}

Ante las grandes tendencias y cambios contemporáneos en el mundo del trabajo, un sentimiento generalizado de "inseguridad" laboral emerge de la percepción de un presente inestable y de un futuro incierto. En este contexto, la psicología del trabajo y de la organización establece como uno de sus focos de atención el impacto psicosocial de la experiencia ocupacional del empleo vinculado al contrato temporal. La actual literatura científica en inglés sobre el tópico se caracteriza por la dispersión terminológica y la escasez de expresiones estandarizadas en el tratamiento del tema. Así, en lo que concierne a la dimensión estructural de la temporalidad del empleo, múltiples sustantivos ("job, employment, work, contract, career, employed, worker"...) son objeto de diversos calificativos ("temporary, unstable, contingent, atypical, non standard"...). Ocasionalmente, se alude directamente a los "temporaries". En cambio, cuando se trata sobre la experiencia subjetiva de esta situación ocupacional, se pone de manifiesto un claro predominio del término "insecurity" acompañando a los de "job", "employment" o "work". Algunas veces, "insecurity" y "unstability" son usados como sinónimos e intercambi-

La investigación incluida en este manuscrito fue parcialmente te subvencionada con cargo al proyecto de investigación SEJ200406680/PSIC, IP Josep M. Blanch. La correspondencia deberá ser enviada al primera autor al Departamento de Psicología Social, Campus de Bellaterra, Universidad Autónoma de Barcelona, 08193 Bellaterra, Cerdanyola del Vallés. E-mail: josepmaria.blanch@uab.es ables; en otras, por el contrario, se presupone que la inseguridad subjetiva deriva de la inestabilidad objetiva, llegando incluso a ser considerada una variable interviniente entre ésta y sus efectos en la salud y el bienestar. Sin embargo, en la mayoría de sus contextos de uso, "insecurity" adquiere un significado más amplio, remitiendo tanto a las características de la situación como a las de la experiencia de la misma. En suma, los tratamientos del tema se distribuyen entre los dos polos de un continuo: el de la clara diferenciación y el de la total confusión de las dos caras de un mismo proceso -la situación de inestabilidad y la percepción de inseguridad-, que suelen aparecer y discurrir conjuntamente.

De Witte (2005) define la "job insecurity" como la "amenaza percibida de pérdida del empleo y las preocupaciones asociadas a la misma". Estudios metaanalíticos y revisiones del tema destacan como características básicas del fenómeno la objetividad del contrato temporal y la subjetividad de la amenaza percibida, el contexto inseguro estresor y la persona insegura estresada, el miedo a un desempleo futurible y el horror a la privación de efectos positivos (financieros y psicosociales) del empleo, los sentimientos de impotencia e indefensión ante malas expectativas ocupacionales, el carácter anticipatorio y prospectivo de emociones asociadas a una pérdida laboral (referida al puesto, a la empresa, al empleo o a la misma posibilidad de trabajar), las diferencias interindividuales en vulnerabilidad 
y en recursos de afrontamiento, la alta incidencia e intensidad de la problemática en el empleo temporal en comparación con el permanente (que, sin embargo, no es inmune a la misma), las connotaciones no sólo laborales sino también sociales y sanitarias de la experiencia, el impacto cuantitativo de sus altas tasas de prevalencia y el cualitativo de su colonización de todos los niveles y sectores sociolaborales; además de su influencia, generalmente negativa, en el funcionamiento psicológico individual y colectivo, en la calidad de vida laboral, en valores, actitudes y conductas concernientes al trabajo y en el clima y la dinámica organizacionales (Auer, 2007, 2008; De Cuyper y De Witte, 2007 a,b, 2008; De Cuyper, Isaksson y De Witte, 2005; De Witte, 1999, 2003, 2005; Hellgren, Sverke e Isakson, 1999; Klandermans y Van Vuuren, 1999; Sverke, Hellgren y Näswall, 2002).

$\mathrm{La}$ investigación ha centrado especialmente el impacto de la "inseguridad" laboral sobre dos ámbitos principales: el "bienestar" y la salud, considerada en sus vertientes física y psicológica (Benach, Benavides, Platt, Díez-Roux, y Muntaner, 2000; Benach, Gimeno y Benavides, 2002; Benavides y Benach, 1999; Benavides, Benach, Díez-Roux y Roman, 2000; Cheng, Chen, Chen, y Chiang. 2005; De Cuyper, Isaksson y De Witte, 2005; De Witte, 2005; Dekker y Schaufeli, 1995; Domenighetti, D'Avanzo y Bisig, 2000; Ferrie, 2001; Ferrie et al, 2001, 2002, 2003, 2005; Hellgren, Sverke e Isaksson, 1999; Hellgren y Sverke, 2003; Kalimo, Taris y Schaufeli, 2003; Lee, Colditz. Berkman y Kawachi, 2004; McDonough, 2000; Mohren, Swaen, Amelsvoort y Galama, 2003;Nolan, Wichert y Burchell, 2000; Sverke y Hellgren, 2002; Silla, Gracia y Peiró, 2005; Sverke, Gallagher y Hellgren, 2000; Sverke, Hellgren y Näswall, 2002; Wichert, Nolan y Burchell, 2000). Sin embargo, la tesis sobre la relación de causalidad de la variable independiente experiencia ocupacional de la temporalidad (integrada por la inestabilidad objetiva del empleo, por la inseguridad percibida del mismo o por la combinación de ambas) sobre la variable dependiente relativa al múltiple impacto negativo no goza del soporte empírico suficiente. El meta-análisis realizado por Sverke, Hellgren y Näswall (2002) pone de manifiesto una enorme variabilidad de los resultados al respecto en cuanto a la magnitud del efecto de la inseguridad laboral sobre el bienestar y especialmente sobre la salud. En algunos estudios, ni tan sólo aparece una asociación significativa entre ambas variables (Sverke y Hellgren, 2002). Estos resultados, atribuidos a la complejidad de la experiencia de la inseguridad laboral y sobre todo a la diversidad y heterogeneidad de la composición del colectivo en situación de empleo temporal, animan el debate sobre cuestiones metodológicas no resueltas en la investigación del tema y sobre deficiencias en el estado actual del conocimiento científico del mismo.

Entre las propuestas de cara a superar estas limitaciones figura la concerniente al desarrollo de la inves- tigación sobre las "variables moderadoras" del proceso, entre las que figuran el apoyo social (Büssing, 1999; Lim, 1996, 1997), el control percibido (Büssing, 1999; De Witte, 2005), la vulnerabilidad individual (Hellgren y Sverke, 2002), la empleabilidad y la justicia percibida (Silla, Gracia y Peiró, 2005) y, muy especialmente, las que se refieren al carácter impuesto o elegido del empleo temporal, con constructos como "preference for contract" (Marler, Barringer y Milkovich, 2002) -que Silla, Gracia y Peiró (2005) operacionalizan mediante una escala monoítem que abarca cinco intervalos de preferencia-, "contract preference" (De Cuyper y De Witte, 2007b), "preferred occupational status" (Chambell y Castanheira, 2007), "willingness to accept a contingent employment" (Bernasek y Kinnear, 2004), "want to be temporaries" (Chambell y Castanheira, 2007), "wish to do temporary employment" (De Cuyper y De Witte, 2007b) y "volition (...) to work on a temporary contract” (De Cuyper y De Witte, 2008).

Ante este panorama disciplinario inmediato y a la vista de ciertos planteamientos políticos de la problemática, la pregunta general que guía la presente investigación está inspirada por un manifiesto del Banco Mundial, en el que esta institución proclama "urbi et orbe" que la desregulación del mercado laboral -matriz de los contratos temporales postfordistas de trabajo- constituye el "mejor" contexto para (que el capital pueda) "hacer negocios" (World Bank, 2007). Puesto que a este mercado concurren diversas partes implicadas, tiene sentido preguntarse además si el trabajo temporal constituye un "buen negocio" para el mismo personal trabajador. Así que la presente investigación constituye un ejercicio más de búsqueda de evidencia empírica sobre los efectos colaterales de la desregulación del trabajo sobre el bienestar laboral.

Con el objetivo general de evaluar el papel moderador de la voluntariedad del contrato laboral de empleo temporal en el impacto de la situación ocupacional sobre el bienestar psicosocial, la estrategia de esta investigación apunta a la construcción y operacionalización de "tipos ideales" de situación ocupacional y a la comparación empírica de sus respectivos niveles de bienestar laboral, controlada la variable "hibridez ocupacional". Atendiendo a la urgencia de clarificar aspectos opacos de la información empírica acumulada sobre el tema, la estrategia metodológica se desarrolla en una dirección relativamente poco considerada por la literatura: la minimización del ruído teórico y empírico que genera un elemento enmascarado y semiinvisible en la reconocida "heterogeneidad" del estatus de empleo temporal en cuanto a composición sociodemográfica y a perfil psicosocial: la "hibridez ocupacional". Tradicionalmente, el conjunto de la población activa ha venido siendo categorizado según variables demográficas o curriculares (género, generación, cualificación, competencias, experiencia, etc.) y estatus ocupacionales convencionales, como empleo (perma- 
nente o temporal) y desempleo (con o sin percepción de subsidio de paro). Estos esquemas clasificatorios, anclados en el sentido común y en el quehacer científico, permiten sistematizar la información con la que organismos como ILO, EUROSTAT o INE siguen dando cuenta de la "perspectiva del empleo" en el mundo, en Europa o en España. Pero el mapa resultante representa, por una parte, unos fenómenos más detectables en el mercado laboral regular que en el universo mercantil paralelo de la economía informal, y por otra, identifica mejor situaciones ya existentes hace tres décadas que otras nuevas, que emergen en un mercado laboral cada día más abierto, complejo y cambiante. En este nuevo estadio del mercado de trabajo, abundan unos perfiles ocupacionales híbridos que se ubican y transitan entre categorías estándar y cuya creciente omnipresencia satura de confusión y borrosidad el panorama ocupacional. Tales perfiles ocupacionalmente híbridos sintetizan, condensan y transmutan características tradicionales del empleo, del desempleo, del subempleo y del no empleo. Por ello, en esta investigación se ha procurado identificar, aislar y excluir, a efectos metodológicos, este cuantitativa y cualitativamente importante segmento "híbrido" de la muestra de población activa estudiada. Mediante este procedimiento, paradójicamente, se ha procedido a trabajar con categorías ocupacionales estándar, pero tratadas sólo como "tipos ideales".

La investigación parte de la doble hipótesis de que la experiencia del empleo temporal involuntario (a) se diferencia significativamente de la que se da en el empleo temporal voluntario y (b) tiene más en común con la experiencia del desempleo que con la del empleo estándar.

\section{Método}

\section{Participantes}

Las personas que participan en la investigación han sido seleccionadas de una muestra de conveniencia de la población activa española, estratificada con criterios de género, generación, situación ocupacional y nivel de cualificación. A sus 769 integrantes se les aplica un cuestionario entre cuyos ítems figuran dos relativos a la propia situación ocupacional actual. Uno de ellos está formulado de acuerdo con un modelo de opción múltiple, ante el que caben las siguientes alternativas de respuesta: (a) empleo por cuenta ajena con contrato indefinido, (b) empleo por cuenta ajena, sin contrato de trabajo, (c) empleo por cuenta ajena con contrato temporal, $(d)$ empleo por cuenta propia, $(e)$ desempleo, $(f)$ estudiando y trabajando, ( $g$ ) otra situación (¿cuál?). El otro consiste en una pregunta abierta con la que invita a detallar de forma más precisa algunas características de la propia situación ocupacional y que proporciona información relevante acerca de si se accedió a la misma voluntariamente o de modo forzado por las circunstancias. De aquel colectivo se extrae 463 personas que pertenecen a alguno de los cuatro siguientes tipos ideales de estatus ocupacional: 1) empleo por cuenta ajena con contrato indefinido, 2) desempleo, 3) empleo temporal voluntario, 4) empleo temporal involuntario. Para la selección de esta muestra final se procede primeramente a identificar tres clases de grupos en función de la autoadscripción en el ítem cerrado: los de "Empleo" (estable), "Empleo Temporal" y "Desempleo". Posteriormente, a partir de la información proporcionada por las respuestas a la pregunta abierta, se confirman (o corrigen) las pertenencias categoriales y se subdivide el grupo "Empleo Temporal" en dos bloques; el "Voluntario" y el "Involuntario".

Al final del proceso, son excluidas, a efectos de esta investigación, 306 personas de la muestra inicial, que constituyen una categoría híbrida y heterogénea en la que concurren, simultánea y habitualmente, fenómenos y procesos tan diversos como el de identificarse como "estudiante" en "desempleo" formal (con carnet de paro), y al tiempo con empleo irregular (sin contrato formal), o con sucesiones de contratos temporales combinados con algunas de las otras situaciones. Y también el personal que combina un "autoempleo" o un "empleo por cuenta ajena" a tiempo parcial y estable (en ámbitos tan diversos como la salud o la construcción) con otro "empleo por cuenta ajena" de carácter "temporal". Y quien "no sabe" dónde autoadscribirse (con los consiguientes conflictos identitarios), ya sea porque cambia muy frecuentemente de situación ocupacional o porque está inscrito oficialmente como "demandante de empleo", pero no para de trabajar en el mercado laboral informal. Tampoco son tenidas en cuenta para el presente estudio las personas que declaran estar autoempleadas o trabajando sin contrato laboral, las que se autopresentan textualmente como "estudiantes" que secundariamente ocupan una parte de su tiempo en actividades laborales y las que, habiendo marcado la casilla correspondiente al empleo temporal, en su respuesta a la pregunta abierta no aportan información suficientemente precisa acerca de si han elegido voluntariamente esta situación ocupacional o si les viene impuesta por falta de alternativas. El concepto de "voluntariedad" que subyace a la distinción entre dos categorías de empleo temporal, el "voluntario" y el "involuntario", se inspira en los de "preferencia", "deseo" y "voluntad" relativos al presente estatus ocupacional (Bernasek y Kinnear, 2004; Chambell y Castanheira, 2007; De Cuyper y De Witte, 2007b, 2008; Marler, Barringer y Milkovich, 2002; Silla, Gracia y Peiró, 2005).

Finalmente, la muestra queda integrada por las siguientes personas, atendiendo a su situación ocupacional: Empleo $(\mathrm{n}=248)$, Desempleo $(\mathrm{n}=106)$, Temporalidad Involuntaria $(n=53)$ y Temporalidad Voluntaria $(n=56)$. La media de edad de la misma es de 31,2 años, tenien- 
do la gran mayoría de sus miembros entre 20 y 45 . Un $60,4 \%$ de los mismos son mujeres. Un tercio de ellos ha cursado estudios primarios, otro tanto terminó los secundarios y el resto tiene o está desarrollando estudios superiores. Cerca del $80 \%$ de sus integrantes reside en el entorno de Barcelona y el otro $20 \%$ se reparte entre Santiago de Compostela y Madrid.

Dentro de esta muestra, y basándonos en las repuestas a las preguntas abiertas, podemos señalar que los estatus de "empleo" y de "desempleo" resultan sociodemográficamente homologables. En cambio, los de empleo temporal "voluntario" e "involuntario" se diferencian en algunos aspectos: la edad media del primer grupo es dos años inferior a la del segundo. Asímismo abunda en el primer grupo, más que en el segundo, el perfil de la persona con titulación universitaria y alta empleabilidad y cualificación, sin cargas familiares, flexible, abierto al cambio y amante de los viajes, entre cuyas prioridades a corto plazo predomina la de "hacer curriculum" sobre la de encontrar pronto "el empleo de su vida"; al que sin embargo no renuncia, a tenor de algunas de sus respuestas a una pregunta abierta sobre cómo visualiza su futuro laboral a largo plazo. También predomina en el primer grupo el joven profesional "flexible" (polivalente, emprendedor y capaz de afrontar riesgos) que, por su alta empleabilidad, no tiene miedo de engrosar las filas del paro. Por el contrario, el grupo de empleo temporal "involuntario" está integrado por un colectivo heterogéneo de personal "flexibilizado": en él aparece en primer lugar el subgrupo con bajos niveles de cualificación y empleabilidad, con cargas familiares y tradicionalmente aferrado al mal menor del empleo precario y temporal. Le acompañan mujeres de todas franjas de edad y hombres jóvenes con escasa experiencia laboral o adultos con "exceso" de la misma. También están representadas en el mismo algunas "minorías" (raciales, étnicas, etc.) y un subgrupo emergente de precariedad laboral posmoderna, integrado por jóvenes valores en los ámbitos de las ciencias, de las letras y de las artes, de la academia y de la sanidad, etc. que -al igual que los miembros pertenecientes a los grupos anteriores- no encuentran el trabajo "decente" (estable) que buscan y merecen.

\section{Instrumento}

Para la recogida de información empírica se ha aplicado de modo individualizado un cuestionario de papel y lápiz que incluye, entre otros elementos, un bloque de preguntas abiertas sobre la actual experiencia ocupacional y sobre el futuro percibido de la misma y una versión abreviada de una escala de Bienestar Laboral (Blanch, 2006), construida con formato de diferencial semántico. Con ella, se trata de evaluar, en un continuo de 0 a 10 , una serie de connotaciones cognitivas y emocionales de la propia experiencia ocupacional y del futuro laboral percibido.
Incluye un listado de 14 ítems bipolares, como los de incertidumbre-certidumbre, malestar-bienestar, pesimismo-optimismo, inseguridad-seguridad, entre otros. Un análisis factorial (componentes principales) revela un único factor que explica un $60.4 \%$ de la varianza, siendo todas las cargas factoriales superiores a .35 y su Alfa de Cronbach de .91 .

\section{Resultados}

En la Tabla 1 aparecen los estadísticos descriptivos (media y desviación típica) en la escala de bienestar laboral de los cuatro grupos utilizados en este estudio. Como puede apreciarse, hay diferencias en las medias de los cuatro grupos, pero no así en las desviaciones típicas. De hecho, puede verse que tanto las personas con empleo estable como aquellas con empleo temporal voluntario puntúan mucho más alta que las personas desempleadas o con empleo temporal involuntario. Clasificando los grupos de acuerdo a su puntuación media, puede verse que los que indican un mayor bienestar son las personas con empleo estable, seguidos por las personas con empleo temporal voluntario, en tercer lugar se sitúan los desempleados y, finalmente, los individuos con empleo temporal involuntario.

Tabla 1. Medias y Desviaciones en la Escala de Bienestar Laboral según Situación Ocupacional

\begin{tabular}{lccr}
\hline Situación Ocupacional & Media & SD & N \\
\hline Empleo Estable & 7.39 & 1.65 & 248 \\
Empleo Temoral Volunatrio & 6.02 & 1.66 & 106 \\
Empleo Temporal Involuntario & 4.50 & 2.09 & 53 \\
Desempleo & 5.09 & 1.97 & 56 \\
\hline
\end{tabular}

Para examinar si dichas diferencias eran estadísticamente significativas realizamos un ANOVA, usando como variable dependiente la puntuación de bienestar laboral y como variable de grupo, los tipos ocupacionales. Los resultados aparecen en la Tabla 2. Puede verse que la variable tipo ocupacional produce diferencias estadísticamente significativas en la escala de bienestar laboral y, atendiendo, al tamaño del efecto, explica un $30 \%$ de la varianza en dicha variable. Por tanto, la hipótesis de partida se confirma. También se realizaron ANOVA con cuatro de los ítems de la escala de bienestar: certidumbre-incertidumbre, seguridadinseguridad, malestar-bienestar y pesimismo-optimismo. Los resultados de dichos ANOVAS aparecen también en la Tabla 2 y, al igual que ocurrió, con la variable dependiente precedente, el tipo ocupacional produce diferencias altamente significativas en todas ellas. Examinando los tamaños de efecto, puede verse que el tipo ocupacional explica un $28.3 \%$ de la variable incertidumbre, un $26.9 \%$ de la inseguridad, un $22.4 \%$ de malestar y un $16.3 \%$ de pesimismo. 
Tabla 2. Resultados de los ANOVA usando el tipo ocupacional como variable independiente

\begin{tabular}{lcccc}
\hline Variable & G.L. & $\mathrm{F}$ & $\mathrm{p}$ & $\eta 2$ \\
\hline Bienestar Laboral & $3 / 459$ & 64.97 & .000 & .298 \\
Incertidumbre & $3 / 459$ & 59.75 & .000 & .283 \\
Inseguridad & $3 / 459$ & 56.00 & .000 & .269 \\
Malestar & $3 / 459$ & 43.86 & .000 & .224 \\
Pesimismo & $3 / 459$ & 28.40 & .000 & .163 \\
\hline
\end{tabular}

Nota. G.L.= Grados de libertad para el numerador y denominador

Para examinar más detenidamente si la condición de voluntariedad en el trabajo temporal produce diferencias, examinamos esta condición particular. Los resultados se muestran en la Tabla 3 e indica que la voluntariedad produce diferencias significativas en las cuatro variables.

Tabla 3. Medias, Desviaciones y Significación de las

Diferencias de Medias por Ítems entre Empleo Temporal "Voluntario" e "Involuntario"

\begin{tabular}{lcccc}
\hline $\begin{array}{l}\text { Situación } \\
\text { Ocupacional }\end{array}$ & $\begin{array}{c}\text { Incertidumbre- } \\
\text { Certidumbre }\end{array}$ & $\begin{array}{c}\text { Inseguridad- } \\
\text { Seguridad }\end{array}$ & $\begin{array}{c}\text { Malestar- } \\
\text { Bienestar }\end{array}$ & $\begin{array}{c}\text { Pesimismo- } \\
\text { Optimismo }\end{array}$ \\
\hline \multirow{2}{*}{ ETV } & 5.30 & 5.86 & 5.77 & 5.98 \\
& $(2.52)$ & $(2.30)$ & $(2.48)$ & $(2.48)$ \\
ETI & 3.13 & 3.45 & 4.17 & 4.52 \\
& $(2.33)$ & $(2.67)$ & $(2.79)$ & $(2.97)$ \\
p & .000 & .000 & .000 & .003 \\
\hline
\end{tabular}

Nota: ETV= Empleo Temporal Voluntario. ETI = Empleo Temporal Involuntario Entre paréntesis la deviación típica y fuera del paréntesis la media.

Realizamos un análisis cualitativo, examinado el contenido de las respuestas a la preguntas abiertas que habíamos incluido en nuestro cuestionario. Las respuestas textuales a las preguntas abiertas concernientes al presente y al futuro percibidos de la propia situación laboral reflejan estos mismos contrastes, de modo que el discurso que emana de la experiencia del Empleo Temporal Involuntario aparece como estrechamente asociado al de la del Desempleo, en tanto que el del Empleo Temporal Voluntario tiende a asociarse al del Empleo Estable. La tabla 4 esquematiza algunas de estas afinidades semánticas.

De los contenidos de esta tabla se desprenden además otras consideraciones: En primer lugar, resulta observable que la inseguridad laboral atraviesa todas las situaciones de empleo, ya que el virus psicológico del miedo a perderlo ha llegado a contagiar el mismo empleo permanente, que, de simplemente "bueno", pasa a a ser considerado "bueno, pero...". También se hace patente que la inseguridad laboral comporta inplanificabilidad, una especie de agujero negro psicosocial que, en el empleo temporal involuntario, deja la panorámica del propio futuro personal y laboral percibido absolutamente a oscuras. De ahí que el malestar asociado a la inseguridad en el empleo temporal invo- luntario incluya un fuerte componente de frustración. Finalmente, puesto que en el desempleo "no hay nada que perder" laboralmente, no resulta sorprendente que algunas personas lo vivan dejando entreabierta una puerta a la "esperanza" de ganar algo en forma de empleo. Cosa que no suele acontecer en el caso del empleo temporal involuntario donde, al predominar el miedo a perder lo (poco) que hay, tienen poca cabida manifestaciones explícitas de esperanza en algo mejor. Ese plus de incertidumbre, desesperanza e inseguridad hace comprensible que, en la escala de bienestar laboral, el estatus de empleo temporal involuntario obtenga una puntuación media más baja que la del mismo desempleo.

\section{Discusión}

La pregunta de investigación sobre la medida y el sentido en que el trabajo temporal contribuye al bienestar del personal trabajador ha encontrado cumplida respuesta en la evidencia empírica de los efectos colaterales del empleo temporal involuntario sobre el bienestar laboral. Los resultados obtenidos en el estudio confirman las dos hipótesis de partida: que la experiencia del empleo temporal involuntario se diferencia significativamente de la que se da en el empleo temporal voluntario y que tiene más en común con la experiencia del desempleo que con la del empleo estándar. Asimismo, en cumplimiento del objetivo general de la investigación, se ha constatado el papel moderador de la voluntariedad del contrato laboral de empleo temporal en el impacto de la situación ocupacional sobre el bienestar psicosocial.

Los datos numéricos expuestos en las tablas son congruentes con la evidencia aportada por otras investigaciones sobre impacto de la inseguridad laboral sobre el bienestar (De Cuyper et al, 2005; De Witte; 2005) y sobre la salud (Benach et al, 2002; Benavides y Benach, 1999; Cheng et al, 2005; Ferrie et al, 2002, 2005; Lee et al, 2004) y sobre el papel moderador de la voluntariedad o preferencia del empleo temporal en la producción de tales efectos (Bernasek y Kinnear, 2004; Chambell y Castanheira, 2007; De Cuyper y De Witte, 2007b, 2008; Silla et al 2005).

Por otra parte, datos textuales como los expuestos en la tabla 4 aportan nuevas evidencias al respecto, señalando que los cambios objetivos en el entorno laboral implican cambios en la subjetivación de la experiencia laboral, especialmente en la perspectiva temporal que conlleva la situación ocupacional. Si el clásico estudio intercultural The Meaning of Working (MOW, 1987) ponía de manifiesto que las expectativas y las metas laborales configuraban una de las dimensiones fundamentales del Significado del Trabajar en la última fase de hegemonía del fordismo, la información cualitativa aportada por esta investigación indica que el empleo temporal involuntario comporta una crisis 
Tabla 4. Presente y futuro ocupacional percibidos en los cuatro tipos ideales de situaciones de empleo

\begin{tabular}{|c|c|c|c|}
\hline & $\begin{array}{l}\text { Presente Situación } \\
\text { Ocupacional }\end{array}$ & Presente Laboral Vivido & Futuro Laboral Percibido \\
\hline 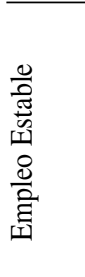 & $\begin{array}{l}\text { BUENA } \\
\text { Excelente, Positiva, } \\
\text { Clara, Normal, } \\
\text { Tranquila, Desafiante } \\
\text { Pero... Inquietante, } \\
\text { Incierta, } \\
\quad \text { Insegura }\end{array}$ & $\begin{array}{l}\text { - "Bien, porque me pagan, me valoran y me relacio- } \\
\text { no...". } \\
\text { - "Sin trabajo, no podría hacer muchas de las cosas } \\
\text { que hago, ni tener muchas cosas que tengo". } \\
\text { - "Económicamente bien, pero termino agotada y } \\
\text { además casi no me queda tiempo para una vida } \\
\text { social y familiar normal". }\end{array}$ & $\begin{array}{l}\text { - "Mi actual situación laboral me posibilita hacer } \\
\text { proyectos de futuro de pareja, de familia, de casa y } \\
\text { de viajes". } \\
\text { - "Aunque ahora no me quejo, espero algo que mejo- } \\
\text { re lo presente" } \\
\text { - "Lo de ahora no sé cuánto me va a durar...". }\end{array}$ \\
\hline 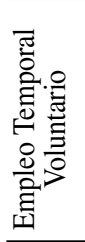 & $\begin{array}{c}\text { NORMAL, } \\
\text { Transitoria, } \\
\text { Buena, } \\
\text { Tranquila, } \\
\text { Por ahora buena...pero... }\end{array}$ & $\begin{array}{l}\text { - "Trabajo y vivo al día, mientras me formo para un } \\
\text { - "Hpleo mejor" } \\
\text { "Hago currículum y experiencia para cuando me } \\
\text { - "Ahora mismo, en el trabajo todo va según mis pla- } \\
\text { nes" }\end{array}$ & $\begin{array}{l}\text { - "Tendré mi trabajo, un trabajo de mi especialidad, } \\
\text { un buen trabajo" ..." } \\
\text { - "Espero trabajar menos, en mejores condiciones y } \\
\text { cobrando más..." }\end{array}$ \\
\hline 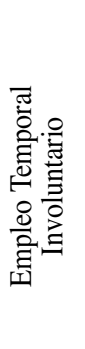 & $\begin{array}{c}\text { MALA, } \\
\text { Difícil, } \\
\text { Complicada, } \\
\text { Incierta, Insegura, } \\
\text { Inestable, Precaria, } \\
\text { Insatisfactoria, } \\
\text { Estresante, } \\
\text { Frustrante, Agobiante, } \\
\text { Desesperante }\end{array}$ & $\begin{array}{l}\text { - "Con mi trabajo, nunca sé, no tengo garantías de } \\
\text { - "Sada, o sea, no tengo nada claro". } \\
\text { - "En estabilidad laboral, nada me funciona bien". } \\
\text { social y familiar normal, no puedo llevar una vida } \\
\text { - "Con trabajo temporal, ni pareja, ni familia, ni } \\
\text { piso, ni coche, ni nada de nada”. }\end{array}$ & $\begin{array}{l}\text { - "Lo veo negativo, oscuro, incierto, inseguro..." } \\
\text { • "No lo veo... No sé... No lo veo bien... No lo veo } \\
\text { claro." } \\
\text { • "Mi situación laboral me impide, pensar y planifi- } \\
\text { car mi futuro. Funciono al día, a corto plazo". } \\
\text { "Años de contrato temporal, con familia, y todo } \\
\text { hipotecado. Esto ya no lo arregla nadie." }\end{array}$ \\
\hline & $\begin{array}{c}\text { NEGATIVA } \\
\text { Confusa, Negra, } \\
\text { Pésima, Complicada, } \\
\text { Crítica, Incierta, } \\
\text { Difícil, Fatal, } \\
\text { Inquietante, } \\
\text { Desesperante, } \\
\text { Pero...con Fe, } \\
\text { Esperanza, Confianza }\end{array}$ & $\begin{array}{l}\text { - "Cuando no tienes trabajo, todo es muy duro, por- } \\
\text { que no ganas para tu familia ni para ti". } \\
\text { - "Sin trabajo, todo va mal"... } \\
\text { - "Mi único "plan" de cada día es encontrar traba- } \\
\text { jo". } \\
\text { - "Esperando encontrar trabajo". }\end{array}$ & $\begin{array}{l}\text { - "Sin trabajo, no puedo hacer planes de vida ni de } \\
\text { nada".- } \\
\text { - -He trabajado muchos años de precario y con con- } \\
\text { tratos temporales. Hace poco, me hicieron fijo. Y } \\
\text { ahora me echaron a la calle en una reducción de } \\
\text { plantilla. Cuando miro al futuro, no veo nada." }\end{array}$ \\
\hline
\end{tabular}

radical en cuanto a expectativas y metas laborales y personales. Las valoraciones de la presente situación ocupacional y del futuro laboral percibido muestran hasta qué punto el trabajo temporal involuntario es una expresión de la "economía de la incertidumbre y de la inseguridad" (Beck,1998), de ese marco de experiencia laboral donde sólo cabe la "certidumbre de la incertidumbre" y la "seguridad de la inseguridad" (Beck, 2000), de la "cultura del nuevo capitalismo" (Sennet, 2006), donde las viejas carreras laborales lineales, continuas, previsibles y programables, fuentes de bienestar personal y de identidad social, se tornan meras sucesiones de anécdotas sin trama ni sentido, proyectos sin plan de viabilidad, trayectorias a la deriva, trágicas biografías rotas (Sennet, 1998) y fracturadas (Beck, 2000).

En el plano metodológico, se ha trabajado con "tipos ideales" de situación ocupacional. Para ello ha sido necesario construir artificialmente una categoría "missing" de casos a excluir de la investigación, ya que su perfil no encaja en ninguno de los tipos establecidos. El conjunto de los casos excluidos constituye el

Revista de Psicología del Trabajo y de las Organizaciones Vol. 25, n. ${ }^{\circ}$ 1, 2009 - Págs. 59-70 colectivo numéricamente más importante de la muestra inicial (un tercio de la misma). Este alejamiento de la representatividad estadística de la muestra con respecto a la población de la que ha sido extraída ha facilitado, en contrapartida, el acercamiento a diferentes categorías puras de experiencia ocupacional y la comparación entre las mismas. De este modo, se ha evitado la confusión empírica y teórica que puede generar la multitud de los casos que desdibujan los contornos categoriales, dificultando la detección de contrastes intergrupales, y acaso dando lugar a resultados contradictorios entre diversas investigaciones. Por otro lado, esta amalgama de casos híbridos da una idea de la complejidad del panorama sociolaboral actual, así como de la progresiva obsolescencia de las categorías convencionales de empleo y desempleo. Estos arcaísmos y sus clásicas definiciones operacionales, saturadas de connotaciones fordistas, subsisten en los informes y memorias de la mayoría de los organismos internacionales competentes en este ámbito. Y son adoptadas además como categorías naturales en no pocos estudios científicos. 
Los resultados presentados en el presente estudio indican (a) que el empleo temporal involuntario comporta no sólo precariedad laboral, sino también psicológica, (b) que el impacto psicológico de esta situación ocupacional tiene más en común con el del desempleo que con el del empleo permanente estándar y (c) que el bienestar laboral en el empleo temporal varía significativamente según si esta situación ocupacional es de carácter voluntario o no. La evidencia persistente de unos efectos psicológicamente aún más devastadores en el empleo temporal involuntario que en el mismo desempleo puede explicarse considerando que la vivencia del desempleo conlleva la certidumbre de "tocar fondo", de "peor imposible" y de que cualquier cambio futurible apuntará "a mejor"; mientras que el "miedo a perder el empleo" bebe de la incertidumbre y retroalimenta la inseguridad percibida.

A la vista de estos efectos colaterales del empleo temporal involuntario en forma de malestar laboral, la tesis del Banco Mundial sobre la desregulación del mercado laboral como el mejor contexto de oportunidad para "doing business" (World Bank, 2007) debe ser matizada: las reformas del mercado laboral que han abierto la puerta a los contratos temporales postfordistas parecen tener un impacto asimétrico, resultando acaso un "negocio" bueno para el accionariado, la gerencia con contrato blindado y el personal central de la organización; pero ciertamente malo para el personal periférico atrapado en un empleo temporal involuntario. En este caso resulta también aplicable el diagnóstico de un libro de reportajes del New York Times (1996) sobre los efectos del "The Downsizing of America": "Workers Fall, Business Rises".

El malestar psicológico en esta situación ocupacional marcada por una inestabilidad temporal indeseada se explica por el contraste entre las experiencias respectivas del empleo estable y del inestable. El empleo permanente con un contrato de duración indefinida facilita una trayectoria laboral lineal, continua, previsible, planificable y controlable, constituyendo un marco de experiencia laboral psicológicamente funcional, en un tiempo caracterizado por la certidumbre y en un espacio que inspira seguridad. En este mundo dominado por la "economía de la certidumbre y de la seguridad" (Beck, 1998), trabajar establemente estructura el tiempo cotidiano, organiza las relaciones sociales, confiere sentido al currículum formativo, a la carrera profesional y al ciclo vital de las personas, funcionando además como una matriz de proyectos, procesos y productos identitarios, mediante los cuales la biografía laboral adquiere una trama continua, coherente y significativa (Jahoda, 1982). Este universo laboral, de signo fordista, en la medida en que es previsible, planificable y controlable, resulta asimismo confortable, agradable y saludable (Warr, 1987). En cambio, en la compleja trama del mercado laboral posfordista, coexisten y se entremezclan situaciones individuales y colectivas de empleo estable con otras marcadas por la temporalidad de los contratos laborales de un personal que los desearía de duración indefinida. Y también abundan en él transiciones del empleo al desempleo y viceversa, así como del empleo temporal al permanente y, sobre todo, en dirección contraria. El desarrollo del empleo temporal involuntario en un tiempo incierto y en un espacio inseguro conlleva el afrontamiento de la impredecibilidad en el plano cognitivo y el de la incontrabilidad en el emocional. Esta situación ocupacional constituye el caldo de cultivo de un personaje trabajador con perfil posmoderno; esto es, de un sujeto débil (Vattimo, 1986), con pensamiento debilitado por la incertidumbre de su destino laboral, dominado por la perplejidad, el desconcierto y el vértigo ante el vacío de un mundo que no le ofrece unas mínimas garantías de seguridad y, por todo ello, estresado, pesimista, insatisfecho, descontento, infeliz, desmotivado y desimplicado con respecto a una organización sólo comprometida con él durante la vigencia del contrato temporal. Sería pues del todo irrazonable e inhumano pedir o esperar de un personal empleado en tales condiciones que, a pesar de todo ello, mostrara señales de bienestar laboral.

El malestar laboral en el empleo temporal involuntario, a pesar de constituir un problema psicológico relevante, permanece socialmente invisible e invisibilizado, porque para el sentido común y para la política convencional, mientras el desempleo aparece como un problema "objetivo" y de cuya existencia "real" no caben dudas, el empleo temporal y la inseguridad que conlleva es considerado un mal menor deseable en la medida en que ahuyenta el fantasma del paro. De ahí que, sus potenciales efectos colaterales de tipo psicológico sean considerados de escasa relevancia social; puesto que no consisten en otra cosa que en un simple "miedo subjetivo" a perder el empleo del que se dispone "objetivamente". Por ello, cualquier intervención práctica sobre la problemática comporta primeramente el reconocimiento de la severidad de la misma y posteriormente la adopción de medidas paliativas y de prevención primaria del problema. El conocimiento acerca del papel de las variables moderadoras de los efectos de la inseguridad laboral (apoyo social, control percibido, empleabilidad, etc.) debe orientar las estrategias de acción en este campo. A este respecto, en la medida en que el malestar asociado a la inseguridad deriva en último término de la incertidumbre, uno de los objetivos de la política preventiva debe consistir en la reduccción de la desinformación, la ambigüedad y la confusión de las que se alimenta esta incertidumbre, mediante la creación o el refuerzo de dispositivos, procesos y contenidos de comunicación y de información.

Sin embargo, acompañando las medidas básicamente paliativas, existen razones para plantear un debate más radical sobre la lógica, la funcionalidad y la misma necesidad de los contratos temporales que, en numerosas ocasiones, sirven para que una misma persona (o una serie de personas distintas) vaya ocupando temporalmente, de modo sucesivo, un mismo puesto 
permanente de trabajo, normalmente en condiciones de precariedad contractual, económica y psicológica. A ese respecto, es pertinente invocar la historia para comprender que los contratos temporales no son un efecto natural del desarrollo económico y laboral, sino de un determinado modo de concebirlo y de gestionarlo. Entraron masivamente en la escena europea durante la "crisis" de los primeros años ochenta, presentados como parte de un paquete de medidas de excepción encaminadas a la "solución" provisional de "problemas" empresariales, presuntamente generados por la tópica "rigidez" de los contratos estables fordistas. Pero de hecho han venido funcionando como expresión de unas "reformas estructurales" que han conducido a una reducción de los costes laborales en un contexto de (neo)liberalización del mercado laboral. Y así, aquellos supuestos remedios para tiempos de crisis han transitado desde el estatus de medidas coyunturales al de imperativos estructurales y casi al de categorías naturales. En el mercado de trabajo español, por ejemplo, han llegado a convertirse en norma estadística (en torno al $80 \%$ ) de los nuevos contratos laborales de los últimos lustros, en un entorno donde durante años la tasa de temporalidad ha rondado el $30 \%$, el doble de la media europea. Una reactualización de la perspectiva histórica de los contratos temporales puede ser un primer paso para evacuarlos definitivamente del escenario del mercado laboral. Esta investigación aporta argumentos para justificar este proceso.

En un balance final de las aportaciones y limita- ciones del presente estudio, destaca en el polo positivo la contundencia de los resultados obtenidos en forma de diferencias significativas entre las cuatro categorías ocupacionales, hecho que refuerza la tesis de la severidad de la problemática psicológica asociada al empleo temporal involuntario y la urgencia de implementar medidas preventivas de la misma. La alta significación estadística de las diferencias intercategoriales observadas es atribuible a la originalidad de la estrategia metodológica adoptada, consistente en trabajar con "tipos ideales" de situación ocupacional, excluyendo un numéricamente importante colectivo "híbrido", que en otros estudios que lo incluyen ha podido generar algo de confusión a la hora de producir e interpretar la información obtenida. En el lado de las limitaciones, figura el tamaño de la muestra, y especialmente el de las categorías de empleo temporal. La rigidez de los criterios de inclusión adoptados generó relativamente pocos problemas a la hora de identificar casos pertenecientes al estatus de "empleo" y al de "desempleo", pero forzó la exclusión técnica de numerosos casos "híbridos" con un pie en el "empleo temporal" regular y el otro en prácticas que contaminan ese estatus (identificarse como "estudiante", estar registrado en el "desempleo", desempeñar simultáneamente un empleo informal, por ejempo). La no inclusión de estos "missing" hace además que en la muestra investigada no quede representada en su heterogeneidad la población activa española. Finalmente, convendría replicar este estudio en otros contextos.

\section{Extended Summary}

Facing the contemporary trends and changes in the world of the work, a generalized feeling of "job insecurity" emerges from the perception of an unstable present and an uncertain future. In this context, the psychology of work and organization focuses its attention on the psycho-social impact of the occupational experience of the temporary work.

The investigation on the "job insecurity" puts in evidence multiple negative collateral effects of this occupational experience, especially in the fields of well-being and health, considered in its physical and psychological dimensions. Nevertheless, an enormous variability of the results as far as the magnitude of the observed effect exists. These results, attributed to the complexity of the experience of job insecurity and mainly to the diversity and heterogeneity of the composition of the group in situation of temporary job, animate the debate on not solved methodological questions in the investigation of the subject and on deficiencies in the present state of the scientific knowledge on this field. Between the proposals facing surpassing these limitations it appears the research on the "moderating variables" of the process, between which they appear the social support, the perceived control, the employability and, very especially, those that talk about to the character imposed or chosen of the temporary job.

With the general objective to evaluate the moderating paper of the wilfulness of the labour contract of temporary job in the impact of the occupational situation on the psycho-social well-being, the strategy of this investigation points at the construction and operationalization of "ideal types" of occupational situation and at the empirical comparison of its respective levels of labour well-being, controlled the variable "occupational hybridiness". Taking care of the urgency to clarify opaque aspects of the accumulated empirical information on the subject, the methodological strategy is developed in a relative address little considered by Literature: the minimisation of the theoretical and empirical noise that generates a masked and semi invisible element in the recognized "heterogeneity" of status of temporary job as far as socio demographic composition and to psycho-social profile: the "occupational hybridiness". 
In an open, complex and changing labour market, every day more, hybrid occupational profiles abound that are located and journeyed between categories standard and whose presence saturates of confusion and fuzziness the occupational panorama. Such personages synthesize, condense and transmute traditional characteristic of the job, unemployment, under employment and the non employment. For this reason, this research aims to identify, to isolate and to exclude, to methodological effects, this quantitative and qualitatively important "hybrid" segment of the sample. The research leaves from the double hypothesis that the experience of the involuntary temporary job (a) difference significantly of which occurs in the voluntary temporary job and (b) is had commoner with the experience of the unemployment that with the one of the standard job.

\section{Method}

\section{Participants}

The people who participate in the investigation have been selected of a sample of convenience of the Spanish working population, stratified with criteria of gender, generation, occupational situation and level of qualification. To its 769 members a questionnaire was applied between whose items they appear two regarding the own present occupational situation. One of them is formulated with a model of multiple options, before which 7 alternatives of answer fit. The other consists of a open question with which it invites to detail of more precise form some characteristics of the own occupational situation and that provides excellent information about if it were acceded to the same voluntarily or of way forced by the circumstances. Of this group it is extracted 463 people who belong to some of the four following ideal types of occupational status: 1) permanent employment, 2) unemployment, 3) voluntary temporary employment, 4) involuntary temporary employment. For the selection of this final sample it is firstly come to identify three classes of groups based on the answers to the closed item: those of permanent employment, unemployment, and temporary employment. Later, from the information provided by the answers to the open question, the categorical properties are confirmed (or correct) and temporary employment in two blocks is subdivided to the group "; the "volunteer" and the "Involuntary" one.

At the end of the process, they are excluded, with the object of this investigation, 306 people of the initial sample, who constitute an hybrid and heterogeneous category in that they concur, simultaneously and habitually, characteristics of more than one occupational situation, or the combination of some of them with the one of "student" or with an informal job.
The sample is integrated by the following people, taking care of its occupational situation: Employment $(n=248)$, Unemployment $(n=106)$, Involuntary Temporary Employment $(n=53)$ and Voluntary Temporary Employment $(n=56)$. The average of age of the same is of 31.2 years, being the great majority of its members between 20 and 45 years old. The $60.4 \%$ of them are women and a third of them finished primary studies, the same finished the secondary ones and the rest finished or is developing high level of studies. Near the $80 \%$ of his members it resides in the surroundings of Barcelona and the other $20 \%$ it is distributed between Santiago de Compostela and Madrid.

\section{Instrument}

For the collection of empirical information it has been applied of individualized way a questionnaire of paper and pencil that it includes, among others elements, a block of open questions on the present occupational experience and on the future perceived of the own labour life, and a been brief version of a scale of Labour Well-being (Blanch, 2006), constructed with semantic differential format. With her, one is to evaluate, in a continuous one of 0 to 10 , a series of mental and emotional connotations of the own occupational experience and of the future labour perceived. It includes a listing of 14 bipolar items, like those of uncertainty-certainty, malaise - well-being, pessimism- optimism, insecurity - security, etc. A factorial analysis (main components) reveals a unique factor that explains $60,4 \%$ of the variance, being all the factorial loads .35 superiors to and their Alpha de Cronbach of .91 .

\section{Results}

They appear significant differences within the sample and between all the categories compose that it as far as average scores in the Scale of Labour Wellbeing. From largest to smallest average score in this scale, the categories appear stepped in the following order: permanent employment, voluntary temporary employment, unemployment and involuntary temporary employment. In the same line, the textual answers to the open questions show a high malaise in status of involuntary temporary employment because the present occupational situation and the uncertainty and insecurity that the own perceived labour future generates.

The contents of these textual answers other considerations are come off in addition: First of all, it is observable that the labour insecurity crosses all the situations of employment, since the psychological virus of the fear to lose it has gotten to infect the same per- 
manent employment, that, of "simply good", happens to a to be considered "good, but...". Also patent is made that the labour insecurity bucket planlessness, a species of psycho-social black hole that, in the involuntary temporary employment, absolutely in the dark leaves the panoramic one of the own perceived personal and labour future. For that reason the malaise associated to the insecurity in the involuntary temporary employment includes a strong component of frustration. Finally, since in unemployment "there is no labour to lose", it is not surprising that to some lives it people leaving half-opened a door the "hope" to gain something in the form of employment. Thing that usually does not occur in the case of the involuntary temporary employment where, when predominating the fear to lose that employment there is, have little capacity explicit manifestations of hope in something better. That extra of uncertainty, hopelessness and insecurity makes comprehensible that, in the scale of labour wellbeing, the involuntary temporary employment status obtains an average score more loss than the one of the same unemployment.

\section{Discussion}

The results obtained in the study confirm the two departure hypotheses: that the experience of the involuntary temporary employment difference significantly of which one occurs in the voluntary temporary employment, and that has commoner with the experience of the unemployment that with the one of the standard employment. Also, the moderating paper of the wilfulness of the labour contract of temporary employment in the impact of the occupational situation has been stated on the psychosocial well-being. These data are congruent with those of other studies. On the other hand, the qualitative information contributed by this investigation indicates that the involuntary temporary employment tolerates a radical crisis concerning labour and personal expectations and goals. The results of the present study indicate (a) that the involuntary temporary employment affects not only labour, but also psychological precarity, (b) that the psychological impact of this occupational situation has the more common with the one of the unemployment that with the one of the standard permanent employment and (c) that the labour well-being in the temporary employment varies significantly according to if this occupational situation it is of voluntary character or no. The persistent evidence of psychological effects still more devastating in the involuntary temporary employment that in the same unemployment can be explained considering that the experience of unemployment entails the certainty of which any change futurible will be better; whereas the "fear to lose the employment" drinks of the uncertainty and feeds back the perceived insecurity.
The labor malaise in the involuntary temporary employment constitutes an urgent challenge not only for psychology, but also for the policy of the work and the organization. Any practical intervention on the problematic one firstly tolerates the recognition of its severity and later the adoption of palliative measures and primary prevention of the problem. The knowledge about the moderating variables of the effects of the labour insecurity (social support, perceived control, employability, etc.) must orient the strategies of action in this field. In this respect, insofar as the malaise associated to the insecurity derives in last term of the uncertainty, one of the objectives of the preventive policy must consist of the reduction of the disinformation, the ambiguity and the confusion on which this uncertainty is fed, by means of the creation or the reinforcement of devices, processes and contents of communication and information. Nevertheless, this investigation contributes arguments to raise a more radical debate on the logic, the functionality and the same necessity of the temporary contracts, that are not a natural effect of the economic development nor an imperative for the sustainability of the companies, but often a simple one half of reduction of the labour costs in a context of neoliberal deregulation of the labour market.

In a final balance of the contributions and limitations of the present study, it emphasizes in the positive pole the forcefulness of the results obtained in the form of significant differences between the four occupational categories, fact that reinforces the thesis of the severity of the psychological problematic associated to the involuntary temporary employment and of the urgency to implement preventive measures of the same. The high statistical significance of the observed inter-categorical differences is attributable to the originality of the adopted methodological strategy, consisting of working with "ideal types" of occupational situation, excluding a numerically important "hybrid" group that may be, in other studies, it has been able to generate something of confusion at the time of producing and processing the obtained data. In the side of the limitations, it appears the sample size, and especially the one of the categories of temporary employment. The rigidity of the adopted criteria of inclusion generated relatively few problems at the time of identifying cases pertaining to status of "permanent employment" and to the one of "unemployment", but it forced the technical exclusion of numerous "hybrid" cases with a foot in the "regular temporary employment" and the other in practices that contaminate that status (to identify themselves like "student", to be registered in "unemployment", simultaneously to carry out an informal job, etc.). The exclusion of these "missing" determines that in the investigated sample is not represented in its heterogeneity the Spanish active population. Finally, this study it would have to be contrasted in other contexts. 


\section{Referencias}

Auer, P. (2008). Auer, P. In search of optimal labour market institutions. Geneva: International Labour Office.

Auer, P. (2007). Security in labour markets: Combining flexibility with security for decent work. Geneva. International Labour Organization.

Beck, U. (1998). World Risk Society. Cambridge: Polity Press.

Beck, U. (2000). The Brave New World of Work. Cambridge: Cambridge University Press.

Benach, J., Benavides, F.G., Platt, S., Díez-Roux, A. y Muntaner, C. (2000) The Health-Damaging Potential of New Types of Flexible Employment: A Challenge for Public Health Researchers. American Journal of Public Health, 90 (8), 1316-17.

Benach, J., Gimeno, D. y Benavides, F.G. (2002). Types of Employment and Health in the European Union. Dublin: European Institute for the Improvement of Living and Working Conditions.

Benavides, F.G. y Benach, J. (1999) Precarious Employment and Health-Related Outcomes in the European Union. Luxembourg: Office for Official Publications of the European Communities.

Benavides, F.G., Benach, J., Díez-Roux, A.V. y Roman, C. (2000). How do Types of Employment Relate to Health Indicators? Findings from the Second European Survey on Work Conditions. Journal of Epidemiology and Community Health 54 (7), 494-501.

Bernasek, A. y Kinnear, D. (2004). Workers' willingness to accept conmtingent employment. Journal of Economic Issues, 33 (2), 461-469.

Blanch, J. M. (2006). Estudio internacional sobre la calidad de la experiencia laboral en el capitalismo flexible. In X. Díaz, L. Godoy, A. Stecher y J. P Toro (Coords.). Trabajo, identidad y Vinculo Social. Reflexiones y experiencias en el capitalismo flexible (pp. 75-107). Santiago de Chile: CEM.

Büssing, A. (1999). Can control at work and social support moderate psychological consequences of job insecurity? Results from a quasi experimental study in the steel industry. European Journal of Work and Organizational Psychology, 8 (2), 219-242.

Chambel, M.J.. y Castanheira, F (2007). They don't want to be temporaries: similarities between temps and core workers. Journal of Organizational Behavior. 28 (8), 943 959.

Cheng, Y., Chen, C.V., Chen, C.J. y Chiangs T.L. (2005). Job insecurity and its association with health among employees in the Taiwanese general population. Social Science \& Medicine. 61 (1), 41.52.

De Cuyper, N. y De Witte, H. (2008).Volition and reasons for accepting temporary employment: Associations with attitudes, well-being, and behavioural intentions. European Journal of Work and Organizational Psychology. 17 (3), 363-387.

De Cuyper, N. y De Witte, H. (2007 a). Job insecurity in temporary versus permanent workers: Associations with attitudes, well-being, and behaviour. Work \& Stress, 21 (1), 65-84.

De Cuyper, N. y De Witte, H. (2007 b). Associations Between Contract Preference and Attitudes, Well-Being and Behavioural Intentions of Temporary Workers. Economic and Industrial Democracy. 28(2): 292 - 312.

De Cuyper, N.; Isaksson, K. y De Witte, H. (Eds). (2005). Employment contracts and well-being among European workers. Aldershot: Ashgate.

De Witte, H. (1999). Job insecurity and psychological wellbeing. Review of the literature and exploration of some unresolved issues. European Journal of Work and Organizational Psychology, 8 (2), 155-177.

De Witte, H. (Ed.) (2005). Job insecurity, union involvement and union activism. Aldershot: Ashgate.

De Witte, H (2005). Job insecurity: Review of the international literature on definitions, prevalence, antecedents and consequences. SA Journal of Industrial Psychology, Vol 31, No 4, 1-6.

De Witte, H. y Näswall, K. (2003). 'Objective' vs 'Subjective' Job Insecurity: Consequences of Temporary Work for Job Satisfaction and Organizational Commitment in Four European Countries. Economic and Industrial Democracy, 24 (2), 149-188.

Dekker, S. y Schaufeli, W. (1995). The effects of job insecurity on psychological health and withdrawal: A longitudinal study. Australian Psychologist, 30 (1), 57-63.

Domenighetti, G., D’Avanzo, B., y Bisig, B. (2000). Health effects of job insecurity among employees in the Swiss general population. International Journal of Health Services, 30(3), 477-490.

Ferrie, J. E. (2001). Is job insecurity harmful to health? Journal of the Royal Society of Medicine, 94 (2), 71-76.

Ferrie, J. E., Shipley, M. J., Marmot, M. G., Martikainen, P., Stansfeld, S. A., y Smith, G. D. (2001). Job insecurity in white-collar workers: toward an explanation of associations with health. Journal of Occupational Health Psychology, 6 (1), 26-42.

Ferrie, J. E., Shipley, M. J., Stansfeld, S. A. y Marmot, M. G. (2002). Effects of chronic job insecurity and change in job security on self-reported health, minor psychiatric morbidity, physiological measures, and health related behaviours in British civil servants: the Whitehall II Study. Journal of Epidemiology and Community Health, 56 (6), 450-454.

Ferrie, J. E., Shipley, M. J., Stansfeld, S.A., Smith, J.D y Marmot, M (2003). Future incertanty and socioeconomic inequalities in health: the Whitehall II study. Social Science \& Medicine. 57 (4), 637-646.

Ferrie, J. E., Shipley, M. J., Newman, K., Stansfeld, S.A. y Marmot, M (2005). Self-reported job insecurity and health in the Whitehall II study: potential explanations of the relationship. Social Science \& Medicine. 60 (7), 1593-1602.

Hellgren, J. y Sverke, M. (2003). Does job insecurity lead to impaired well-being or vice versa? Estimation of crosslagged effects using latent variable modelling. Journal of Organizational Behavior, 24, 215-236.

Hellgren, J., Sverke, M., y Isaksson, K. (1999). A two- 
dimensional approach to job insecurity: Consequences for employee attitudes and well-being. European Journal of Work and Organizational Psychology, 8 (2), 179-195.

Jahoda, M. (1982). Empleo y Desempleo: un análisis sociopsicológico. Madrid. Morata. 1987.

Kalimo, R., Taris, T. W., y Schaufeli, W. B. (2003). The Effects of Past and Anticipated Future Downsizing on Survivor Well-Being: An Equity Perspective. Journal of Occupational Health Psychology, 8 (2), 91-109.

Klandermans, B. y Van Vuuren, T. (1999). Job insecurity. European Journal of Work and Organizational Psychology, 8 (2), 145-314.

Lee, S., Colditz. G.A., Berkman, L.F. y Kawachi, I (2004). Prospective study of job insecurity and coronary heart disease in US women. Annals of Epidemiology. 14, (1), 24-30.

Lim, V. (1996). Job insecurity and its outcomes: Moderating effects of work-based and non work-based social support. Human Relations, 49 (2), 171-194.

Lim, V. (1997). Moderating effects of work-based support on the relationship between job insecurity and its consequences. Work \& Stress, 11 (3), 251-266.

Marler, J., Barringer, M. V. y Milkovich, G. T. (2002). Boundaryless and Traditional Contingent Employees: Worlds apart. Journal of Organizational Behavior, 23, 425.453 .

McDonough, P. (2000). Job insecurity and health. International Journal of Health Services, 30(3), 453-476.

Mohren, D. C., Swaen, G. M., van Amelsvoort, L. G., Borm, P. J. y Galama, J. M. (2003). Job insecurity as a risk factor for common infections and health complaints. Journal of Occupational and Environmental Medicine, 45(2), 123-129.

MOW. International Research Group. (1987). The Meaning of Working. London: Academic Press.

Näswall, K. y De Witte, H. (2003). Who feels insecure in Europe? Predicting job insecurity from background variables. Economic and Industrial Democracy, 24 (2), 189215.

New York Times (1996). The Downsizing of America. New York. Times Books-Random House.
Nolan, J., Wichert, I. y Burchell, B. (2000). Job insecurity, psychological well-being and family life. In E. Heery y J. Salmon (Eds.). The insecure workforce (pp. 181-209). London: Routledge.

Sennet, R. (2006). The Culture of the New Capitalism New Haven: Yale University Press.

Sennett, R. (1998). The Corrosion of Character. The Personal Consequences Of Work In the New Capitalism. New York: Norton.

Silla, I., Gracia, R. F. y Peiró, J. M (2005). Job Insecurity and Health-Related Outcomes among Different Types of Temporary Workers. Economic and Industrial Democracy, 26 (1), 89-117.

Sverke, M., Gallagher, D. G., y Hellgren, J. (2000). Alternative work arrangements: Job stress, well-being and pro-organizational attitudes among employees with different employment contracts. In K. Isaksson, C. Hogstedt, C. Eriksson, \& T. Theorell (Eds.), Health effects of the new labour market (pp. 145-167). New York: Plenum.

Sverke, M. y Hellgren, J. (2002). The nature of job insecurity: Understanding employment insecurity on the brink of a new millennium. Applied Psychology: An International Review, 51 (1), 23-42.

Sverke, M., Hellgren, J. y Näswall, K. (2002). No security: A meta-analysis and review of job insecurity and its consequences. Journal of Occupational Health Psychology, 7 (3): 242-264.

Sverke, M., Hellgren, J., Näswall, K., Chirumbolo, A., De Witte, H. y Goslinga, S. (2004). ob insecurity and union membership. European unions in the wake of flexible production. Brussels: P.I.E.-Peter Lang.

Vattimo, G. (1986). El fin de la modernidad. Barcelona: Gedisa.

Warr, P. (1987). Work, Unemployment and Mental Health. Oxford, UK: Oxford University Press.

Wichert, I.C., Nolan, J.P. y Burchell, B. J. (2000). Workers on the Edge: Job Insecurity, Psychological wellbeing and Family Life. Washington DC: Economic Policy Institute.

World Bank (2007). Doing Business in 2008. Washington DC: The World Bank.

Manuscrito Recibido: 20/1/2009

Revisión Recibida: 24/3/2009

Aceptado: 26/3/2009 